\title{
Consensus of the Second-order Multi-agent Systems under Asynchronous Switching with a Controller Fault
}

\author{
Dianhao Zheng*, Hongbin Zhang, J. Andrew Zhang and Yang Li
}

\begin{abstract}
Asynchronous switching differing from asynchronous consensus may hinder the system to reach a consensus. This receives very limited attention, especially when the multi-agent systems have a controller fault. In order to analyze the consensus in this situation, this paper studies the consensus of the second-order multi-agent systems under asynchronous switching with a controller fault. We convert the consensus problems under asynchronous switching into stability problems and obtain important results for consensus with the aid of linear matrix inequalities. An example is given to illustrate the effect of asynchronous switching on the consensus, and to validate the analytical results in this paper.
\end{abstract}

Keywords: Consensus, Multi-agent systems, Asynchronous switching, Controller faults

\section{INTRODUCTION}

Multi-agent systems, as a class of complicated dynamic systems, are composed of multiple interactive intelligences. Such systems appear in many applications, such as robot communication networks [1,2], and unmanned vehicles [3]. Therefore multi-agent systems have drawn considerable attention in recent years [4-8]. In multiagent systems, all agents coordinate to solve a global common problem, such as consensus [4], containment control $[9,10]$, and vehicle formations.

Consensus, as the most basic problem of the coordination in multi-agent systems, is to design or analyze a distributed control law to make all agents reach a common value [11]. Whenever the nodes of a network are all in agreement, this common value is called the group decision value. Reaching the group decision value needs to apply inputs that only depend on the states of every node and its own neighbours in a distributed way [4].

The consensus problem has been extensively studied. Some basic concepts on consensus and topologies were introduced in $[4,12]$. In [13], results for second-order consensus under fixed topologies are presented. Second-order consensus under switched topologies was studied in [14]. The paper [11] studied the consensus problem for a class of uncertain multi-agent systems under directed switch- ing networks with uncertainty. The papers $[15,16]$ studied the consensus problem for a class of general second-order multi-agent systems and presented some results about the necessary and sufficient condition for the consensus. Fixed-time consensus tracking control for second-order multi-agent systems with bounded input uncertainties was introduced in [17]. A distributed protocol was proposed in [18] based on the information of second-order neighbours for the robust consensus problem of fractional-order linear multi-agent systems with positive real uncertainty under a fixed undirected topology.

So far, almost all the research efforts on the consensus of multi-agent under switched topologies are devoted to networks without feedback control or with only synchronous switching despite the great importance of the consensus under asynchronous switching control in both theoretical and practical aspects [19]. According to the definition in [20-22], the consensus is called asynchronous consensus if the individuals in systems respond to the new information from their neighbours at different update times. This is different to the consensus under asynchronous switching, which means that the switching between the candidate controllers and topologies are asynchronous [19]. For switched systems, the asynchronous switching often means that the switching of the controllers

This work is supported by the National Natural Science Foundation of China( Grant No.61374117)

Dianhao Zheng is with the School of Information and Communication Engineering, University of Electronic Science and Technology of China, Chengdu 611731, China, is also with the Faculty of Engineering and Information Technology, University of Technology Sydney, Sydney, NSW 2007, Australia (e-mail: dianhao18@126.com). Hongbin Zhang is with the School of Information and Communication, University of Electronic Science and Technology of China, Chengdu 611731, China (e-mail:zhanghb@uestc.edu.cn). J. Andrew Zhang is with the Global Big Data Technologies Centre, University of Technology Sydney, Sydney, NSW 2007, Australia (e-mail:Andrew.Zhang@uts.edu.au). Yang $\mathrm{Li}$ is with the School of Information and Communication, University of Electronic Science and Technology of China, Chengdu, Sichuan, 611731, China. (e-mail:13648065067@163.com).

* Corresponding author. 
to be designed has a lag to the switching of the system models [23] and the switching between the candidate controllers and system modes is asynchronous [24]. Therefore, although asynchronous consensus and consensus under asynchronous switching sound similar in their names, their definitions are different and asynchronous switching may hinder the way to a consensus.

Asynchronous switching has been studied for switched systems in the past few years and the research results on switched systems have advanced the study on switched topologies [23-25]. Due to link failures or reconnection, the topologies of multi-agent systems often change. When the changing is modelled in a switched way, the multi-agent systems under switching topologies can be described and analyzed by switched systems theorems [24]. However, the consensus problem for asynchronous switching is yet to be solved. Since asynchronous switching widely exists in switched topologies, the consensus of multi-agent systems under asynchronous switching needs to be investigated urgently, especially for the systems with a controller fault.

The main purpose of this paper is to study the consensus of the second-order multi-agent systems under asynchronous switching with a controller fault. We will tackle the problem via converting the consensus problems under asynchronous switching into stability problems. The rest of the paper is organized as follows. In Section 2, some basic concepts and algebraic graph theories are given. Main results about asynchronous switching are presented in Section 3. The simulation results are presented in Section 4. Finally, conclusions are provided in Section 5.

Notations: The superscript " $T$ " stands for matrix transposition. The mathematical symbols $I_{n-1}$ and $0_{n-1}$ mean an identity matrix and a zero matrix with $(n-1) \times(n-1)$ dimension, respectively. The sign $\operatorname{diag}\{\cdots\}$ represents a block-diagonal matrix with proper dimension. If $P$ is a given matrix, $P>($ or $<) 0$ signifies a symmetric and positive (or negative) definite matrix $P$. For a function $\gamma$, it is said to be of a class $K_{\infty}$ function if the function $\gamma:[0, \infty) \rightarrow[0, \infty), \gamma(0)=0$, is strictly increasing, continuous, and unbounded. Scalar multiplication of matrices is defined as a regular number( called a "scalar" ) multiplying every element in the matrix [26].

\section{PRELIMINARIES}

In this section, some basic concepts and algebraic graph theories are introduced.

The network of multi-agent systems is often modelled by graph theories. For the multi-agent systems with $n$ agents and the node indexes set $\mathcal{I}=\{1,2, \ldots, n\}$, the digraph can be denoted by $\mathcal{G}=(\mathcal{V}, \mathcal{E}, \mathcal{A})$, where $\mathcal{V}=$ $\left\{v_{1}, v_{2}, \ldots, v_{n}\right\}, \mathcal{E} \subseteq \mathcal{V} \times \mathcal{V}, \mathcal{A}=\left[a_{i j}\right]$ are the set of nodes, the set of edges and the adjacency matrix. The edge between nodes $i$ and $j$ can be described as $e_{i j}=\left(v_{i}, v_{j}\right)$. This method can represent any edge of the graph $\mathcal{G}$. We assume $i \neq j$ for any edge. The set of neighbours of node $v_{i}$ is denoted by $\mathcal{N}_{i}=\left\{v_{j} \in \mathcal{V}:\left(v_{i}, v_{j}\right) \in \mathcal{E}, j \neq i\right\}$. The Laplacian matrix $L$ is defined as: $l_{i j}=\sum_{k=1, k \neq i}^{n} a_{i k}$ for $i=j$, and $l_{i j}=-a_{i j}$, for $i \neq j, i, j \in \mathcal{I}$. A Laplacian-like matrix $H$ is defined as $H=\left[h_{i j}\right]$, where $h_{i j}=l_{i j}-l_{n j}$. The function $\sigma(t):[0,+\infty) \rightarrow \mathcal{M}=\{1,2, \cdots, m\}$ stands for the switching signal of the switching topologies, where $m$ is the total amount of topologies. The function $\sigma^{\prime}(t)$ denotes the asynchronous switching of $\sigma(t)$.

For the switched topologies, $t_{1}, t_{2}, t_{3}, \cdots, t_{l}, t_{l+1}, \cdots$ stand for the switching times of the topologies of the multi-agent systems. Let $\Delta_{\sigma\left(t_{l}\right)}\left(t_{l}, t_{l+1}\right)\left(\right.$ or $\nabla_{\sigma\left(t_{l}\right)}\left(t_{l}, t_{l+1}\right)$ ) represent the asynchronous (or synchronous) time between the time slots $\left[t_{l}, t_{l+1}\right)$. The symbol $T_{p}(0, t)$ denotes the running time of the $p^{t h}$ topology between the time slots $[0, t)$.

For a group of $n$ agents systems, every agent is modelled by the second-order dynamics as

$$
\begin{aligned}
& \dot{x}_{i}(t)=v_{i}(t), \\
& \dot{v}_{i}(t)=u_{i}(t),
\end{aligned}
$$

where $x_{i}(t)$ is the state of the $i^{t h}$ agent, $u_{i}$ is the control input.

For asynchronous switching, there exists a time lag $\Delta$ between the topologies switching signal $\sigma(t)$ and the feedback controller coefficients switching $\sigma^{\prime}(t)$. When the lag is small enough, the models tend to be synchronous. Using one of the many algorithms for synchronous switching $[27,28]$ as an example, the corresponding consensus algorithm with asynchronous switching can be represented as

$$
\begin{aligned}
& u_{i}(t) \\
& =f \beta_{0_{\sigma(t-\Delta)}} \sum_{j \in \mathcal{N}_{\mathrm{i}}(\mathrm{t})} a_{i j_{\sigma(t)}}\left(x_{j}(t)-x_{i}(t)\right)-f \beta_{1_{\sigma(t-\Delta)}} v_{i}(t),
\end{aligned}
$$

where $a_{i j_{\sigma(t)}}, i, j \in \mathcal{I}$ are the elements in the adjacency matrix $\mathcal{A}(\mathcal{G})$. The topology $\mathcal{G}$ and $\sigma(t)$ change at the switching times $t_{1}, t_{2}, t_{3}, \cdots, t_{l}, t_{l+1}, \cdots$. The positive parameters $\beta_{0}, \beta_{1}$ are the coefficients, and $f$ is defined as

$$
f \triangleq \frac{\text { measured value }}{\text { actual value }} \text {. }
$$

The fault considered in this model is uncertain and

$$
\left\{\begin{array}{c}
0<f_{d} \leq f \leq f_{u}, \\
f_{d} \leq 1 \leq f_{u},
\end{array}\right.
$$

where $f_{d}$ and $f_{u}$ are known constants. In this paper, we only assume the range of the deviation is known.

Remark 1: When the positive constants $f_{d} \neq 1$ or $f_{u} \neq 1$ are known, it is implied that consensus feedback has an uncertain parameter $f$. It may originate from inaccurate coefficient $\beta$ or other factors and results in a controller fault. 
Remark 2: Because of $\Delta$ in the system (2), the switching time of topologies and controllers is different and the system (2) is under asynchronous switching. Since all agents update their states at the same time $t$, it is also a synchronous system. Therefore, the system (2) is a synchronous system with asynchronous switching. For asynchronous consensus, every agent has its own update time $t_{i}$, which is different from agent to agent. For the system (2), the controller could be at fault and the parameters could be uncertain and mismatched. How to reach a consensus in this situation is challenging.

For all initial conditions, the multi-agent systems (1) are said to reach a consensus if

$$
\lim _{t \rightarrow+\infty}\left[x_{i}-x_{j}\right]=0, \lim _{t \rightarrow+\infty}\left[v_{i}-v_{j}\right]=0, \forall i, j \in \mathcal{M}, i \neq j .
$$

For a switching $\sigma(t)$ at any time $t_{k}>t_{l} \geq 0$, the switching number in the $p^{t h}$ subsystem is denoted by $N_{\sigma p}\left(t_{l}, t_{k}\right)$, and the total running time of $p^{\text {th }}$ subsystem is denoted by $T_{p}\left(t_{l}, t_{k}\right)$ over the interval $\left[t_{l}, t_{k}\right)$. In [29], it is shown that the system has a mode-dependent average dwell time (MDADT) $\tau_{a p}$ if there exist positive numbers $N_{0 p}\left(t_{l}, t_{k}\right)$ and $\tau_{a p}$ such that

$$
N_{\sigma p}\left(t_{l}, t_{k}\right) \leq N_{0 p}\left(t_{l}, t_{k}\right)+\frac{T_{p}\left(t_{l}, t_{k}\right)}{\tau_{a p}}
$$

Based on the concept on MDADT, we can get the following Lemma 1.

Lemma 1: Consider a system $\dot{z}_{t}=A_{\sigma(t-\Delta)} z_{t}, \sigma(t-$ $\Delta) \in \mathcal{M}$ with give constants $\lambda_{p}>0, \mu_{p}>1, \alpha_{p}>0, p \in$ $\mathcal{M}$. For $\forall p \in \mathcal{M}, \forall t \in\left[t_{l}, t_{l+1}\right)$, and $\forall\left(\sigma\left(t_{l}\right)=p, \sigma\left(t_{l}^{-}\right)=\right.$ $q) \in \mathcal{M} \times \mathcal{M}, p \neq q$, if there exist symmetric matrices $P_{p}>0$, such that

$$
\begin{array}{r}
A_{q}^{T} P_{p}+P_{p} A_{q} \leq \alpha_{p} P_{p}, \forall t \in\left[t_{l}, t_{l}+\Delta_{l}\right), \\
A_{p}^{T} P_{p}+P_{p} A_{p} \leq-\lambda_{p} P_{p}, \forall t \in\left[t_{l}+\Delta_{l}, t_{l+1}\right) \\
\text { and } P_{p}\left(z\left(t_{l}\right)\right) \leq \mu_{p} P_{q}\left(z\left(t_{l}^{-}\right)\right),
\end{array}
$$

then the system is globally uniformly asymptotically stable with MDADT

$$
\tau_{a p}>\tau_{a p}^{*} \triangleq \frac{\Delta_{p_{-} \max }\left(\lambda_{P}+\alpha_{p}\right)+\ln \mu_{p}}{\lambda_{p}},
$$

where $\Delta_{p_{-} \max } \triangleq \max _{l, \sigma\left(t_{l}\right)=p} \Delta_{\sigma\left(t_{l}\right)}\left[t_{l}, t_{l+1}\right)$, for $\forall l \in \mathbb{N}$.

Proof: For any $t>0, \forall t \in\left(t_{l}, t_{l+1}\right)$.

From (8), one has

$$
\frac{\ln \mu_{p}+\left(\lambda_{p}+\alpha_{p}\right) \Delta_{p_{-} \max }}{\tau_{a p}}-\lambda_{p}<0,
$$

and

$$
\max _{p \in \mathcal{M}}\left\{\frac{\ln \mu_{p}+\left(\lambda_{p}+\alpha_{p}\right) \Delta_{p_{-} \max }}{\tau_{a p}}-\lambda_{p}\right\}<0 .
$$

A multiple Lyapunov function is constructed as

$$
V_{p}(z(t))=z(t)^{T} P_{p} z(t) .
$$

According to (5), (6) and (7), it holds that

$$
\begin{aligned}
& V_{\sigma(t)}(x(t)) \\
\leq & \exp \left\{\alpha_{\sigma\left(t_{l}\right)} \Delta_{\sigma\left(t_{l}\right)}\left(t_{l}, t\right)-\lambda_{\sigma\left(t_{l}\right)} \nabla_{\sigma\left(t_{l}\right)}\left(t_{l}, t\right)\right\} V_{\sigma\left(t_{l}\right)}\left(x\left(t_{l}\right)\right) \\
\leq & \exp \left\{\alpha_{\sigma\left(t_{l}\right)} \Delta_{\sigma\left(t_{l}\right)}\left(t_{l}, t\right)-\lambda_{\sigma\left(t_{l}\right)} \nabla_{\sigma\left(t_{l}\right)}\left(t_{l}, t\right)\right\} \\
& \times \mu_{\sigma\left(t_{l}\right)} V_{\sigma\left(t_{l}\right)}\left(x\left(t_{l}{ }^{-}\right)\right) \\
\cdots & \ldots \\
\leq & \left\{\prod_{p=1}^{l} \mu_{\sigma\left(t_{p}\right)}\right\} \\
& \times \exp \left\{\alpha_{\sigma\left(t_{l}\right)} \Delta_{\sigma\left(t_{l}\right)}\left(t_{l}, t\right)+\cdots+\alpha_{\sigma\left(t_{0}\right)} \Delta_{\sigma\left(t_{0}\right)}\left(t_{l-1}, t_{l}\right)\right\} \\
& \times \exp \left\{-\lambda_{\sigma\left(t_{l}\right)} \nabla_{\sigma\left(t_{l}\right)}\left(t_{l}, t\right)-\cdots-\lambda_{\sigma\left(t_{0}\right)} \nabla_{\sigma\left(t_{0}\right)}\left(t_{0}, t_{1}\right)\right\} \\
& \times V_{\sigma\left(t_{0}\right)}\left(x\left(t_{0}\right)\right) .
\end{aligned}
$$

Because of the total switching numbers $N(0, t)=$ $\sum_{p=1}^{m} N_{\sigma p}(0, t)$ and $t_{0}=0$, one can get

$$
\begin{aligned}
& V_{\sigma(t)}(x(t)) \\
\leq & \left\{\prod_{p=1}^{m} \mu_{p}^{N_{\sigma p}(0, t)}\right\} \exp \left\{\sum_{p=1}^{m} \alpha_{p} \Delta_{p}(0, t)-\lambda_{p} \nabla_{p}(0, t)\right\} \\
& \times V_{\sigma(0)}(x(0)) .
\end{aligned}
$$

According to (4), one has

$$
\begin{aligned}
& \prod_{p=1}^{m} \mu_{p}^{N_{\sigma_{p}}(0, t)}=\prod_{p=1}^{m} \mu_{p}^{N_{0 p}+\frac{T_{p}(0, t)}{\tau_{a p}}} \\
= & \exp \left\{\sum_{p=1}^{m}\left\{N_{0 p} \ln \mu_{p}+\frac{T_{p}(0, t)}{\tau_{a p}} \ln \mu_{p}\right\}\right\}
\end{aligned}
$$

and

$$
\begin{aligned}
& V_{\sigma(t)}(x(t)) \\
\leq & \exp \left\{\sum_{p=1}^{m}\left\{N_{0 p} \ln \mu_{p}+\frac{T_{p}(0, t)}{\tau_{a p}} \ln \mu_{p}\right\}\right\} \\
& \times \exp \left\{\sum_{p=1}^{m}\left\{\alpha_{p} \Delta_{p}(0, t)-\lambda_{p} T_{p}(0, t)+\lambda_{p} \Delta_{p}(0, t)\right\}\right\} \\
& \times V_{\sigma(0)}(x(0)) \\
\leq & \exp \left\{\sum_{p=1}^{m} N_{0 p} \ln \mu_{p}+\left(\frac{\ln \mu_{p}}{\tau_{a p}}-\lambda_{p}\right) T_{p}(0, t)\right\} \\
& \times \exp \left\{\sum_{p=1}^{m}\left(\lambda_{p}+\alpha_{p}\right) \Delta_{p}(0, t)\right\} V_{\sigma(0)}(x(0)) .
\end{aligned}
$$

From the definition of $\Delta_{p_{-}} \max$ one can get

$$
\Delta_{p}(0, t) \leq N_{\sigma p}(0, t) \Delta_{p_{-} \max } \leq N_{0 p}+\frac{T_{p}(0, t)}{\tau_{a p}} .
$$


Then, based on (4), one can get

$$
\begin{aligned}
& V_{\sigma(t)}(x(t)) \\
\leq & \left.\exp \left\{\sum_{p=1}^{m}\left\{N_{0 p} \ln \mu_{p}+\left(\lambda_{p}+\alpha_{p}\right) N_{0 p} \Delta_{p_{-} \max }\right)\right\}\right\} \\
& \times \exp \left\{\sum_{p=1}^{m}\left\{\left(\frac{\ln \mu_{p}+\left(\lambda_{p}+\alpha_{p}\right) \tau_{a p}}{\tau_{a p}}-\lambda_{p}\right)\right\} T_{p}(0, t)\right\} \\
& \times V_{\sigma(0)}(x(0)) .
\end{aligned}
$$

From (9), we can conclude that $V_{\sigma(t)}(x(t))$ convergences to zero, as $t \rightarrow+\infty$, if the dwell time satisfies $\left.\tau_{a p}>\left(\lambda_{p}+\alpha_{p}\right) \Delta_{p_{-} \max }+\ln \mu_{p}\right) / \lambda_{p}$.

Therefore, we can see that the asymptotic stability is reduced. This completes the proof.

In order to solve the problems with an uncertain parameter, the following Lemma 2 is introduced.

Lemma 2: [30] Given matrices $Q, R, E$ and $H$ with proper dimensions, and $Q=Q^{T}, R=R^{T}>0$, the inequality

$$
Q+H F E+E^{T} F^{T} H^{T}<0
$$

holds for all $F$ satisfying $F^{T} F \leq R$, if and only if there exists some $\varepsilon>0$, such that

$$
Q+\varepsilon H H^{T}+\varepsilon^{-1} E^{T} R E<0 .
$$

\section{MAIN RESULTS}

In this section, we mainly complete the following three objectives:

Firstly, we design a transformation from consensus under asynchronous switching to stability. For asynchronous switching, the states of agents may go to divergences when some necessary and sufficient conditions cannot be met [15] in the asynchronous switching time slots. We analyze the relationship between consensus under asynchronous switching and the asynchronous stability of switched systems and then present the conversion between them. Based on the conversion and the relationship between energy functions and stability, the divergences will cause the increase of the energy functions. That is, asynchronous switching may result in the divergences of states, which are marked by the increasing of the energy function.

Secondly, we analyze the consensus with a controller fault. We rewrite the formation of $f$ and analyze the conditions for reaching a consensus when the multi-agent systems are switching with a controller fault based on the method of the mode-dependent average dwell time. Using this method, we can decrease the overall energy function by adjusting the dwell time of topologies, and hence force the system to reach a consensus.

Thirdly, we extend the results on asynchronous switching to the synchronous switching.

\subsection{Transformation from Consensus to Asyn- chronous Switching}

By the following Theorem 1, the problem of the consensus under asynchronous switching is converted into that of the asynchronous stability of switched systems.

Theorem 1: For the multi-agent systems with directed network $\mathcal{G}(t)$, if the system

$$
\dot{z}(t)=F K_{\sigma(t-\Delta)} B_{a \sigma(t)} z(t), z\left(t_{0}\right)=z(0),
$$

is globally uniformly asymptotically stable, then the multi-agent systems with the dynamic system (1) will reach a consensus with the consensus algorithm (2), where

$$
\begin{aligned}
& F=\left[\begin{array}{ll}
I_{n-1} & 0_{n-1} \\
0_{n-1} & f_{\text {diag }}
\end{array}\right], \\
& K_{\sigma(t-\Delta)}=\left[\begin{array}{cc}
0_{n-1} & I_{n-1} \\
-\beta_{0} I_{n-1} & -\beta_{1} I_{n-1}
\end{array}\right]_{\sigma(t-\Delta)}, \\
& B_{a \sigma(t)}=\left[\begin{array}{cc}
H & 0_{n-1} \\
0_{n-1} & I_{n-1}
\end{array}\right]_{\sigma(t)}, \\
& f_{\text {diag }}=\operatorname{diag}\{\underbrace{f, \cdots, f}_{n-1}\} .
\end{aligned}
$$

Proof: Firstly, we define $z(t) \triangleq\left[x_{1}-x_{n}, \ldots, x_{n-1}-\right.$ $\left.x_{n}, v_{1}-v_{n}, \ldots, v_{n-1}-v_{n}\right]^{T}(t)$.

According to the definition of the consensus, if $\lim z(t)=0$, the multi-agent systems will reach a con$t \rightarrow+\infty$
sensus.

From (2), one can get

$$
\begin{aligned}
& u_{i}-u_{n} \\
= & f \beta_{0_{\sigma(t-\Delta)}} \sum_{j \in \mathcal{N}_{\mathrm{i}}(\mathrm{t})} a_{i j_{\sigma(t)}}\left(x_{j}(t)-x_{i}(t)\right)-f \beta_{1_{\sigma(t-\Delta)}} v_{i}(t) \\
& -f \beta_{0_{\sigma(t-\Delta)}} \sum_{k \in \mathcal{N}_{\mathrm{n}}(\mathrm{t})} a_{n k_{\sigma(t)}}\left(x_{k}(t)-x_{n}(t)\right)+f \beta_{1_{\sigma(t-\Delta)}} v_{n}(t) \\
= & -f \beta_{0_{\sigma(t-\Delta)}} L_{i}(t) x(t)-\beta_{1_{\sigma(t-\Delta)}} f v_{i}(t) \\
& +f \beta_{0_{\sigma(t-\Delta)}} L_{n}(t) x(t)+\beta_{1_{\sigma(t-\Delta)}} f v_{n}(t) \\
= & -f \beta_{0_{\sigma(t-\Delta)}}\left(L_{i}(t)-L_{n}(t)\right) x(t)-f \beta_{1_{\sigma(t-\Delta)}}\left(v_{i}(t)-v_{n}(t)\right) \\
= & -f \beta_{0_{\sigma(t-\Delta)}} H_{i}(t)\left[x_{1}-x_{n}, \ldots, x_{n-1}-x_{n}\right]^{T} \\
& -f \beta_{1_{\sigma(t-\Delta)}}\left(v_{i}(t)-v_{n}(t)\right)
\end{aligned}
$$

and

$$
\begin{aligned}
& {\left[\dot{v}_{1}-\dot{v}_{n}, \ldots, \dot{v}_{n-1}-\dot{v}_{n}\right]^{T}(t) } \\
= & {\left[u_{1}-u_{n}, \ldots, u_{n-1}-u_{n}\right]^{T} } \\
= & -f \beta_{0_{\sigma(t-\Delta)}} H(t)\left[x_{1}-x_{n}, \ldots, x_{n-1}-x_{n}\right]^{T}(t) \\
& -f \beta_{1_{\sigma(t-\Delta)}}\left[v_{1}-v_{n}, \ldots, v_{n-1}-v_{n}\right]^{T}(t) \\
= & {\left[-f \beta_{0_{\sigma(t-\Delta)}} H(t),-f \beta_{1_{\sigma(t-\Delta)}} I\right] z(t) . }
\end{aligned}
$$


where $L_{i}$ ( or $H_{i}$ ) is the $i^{\text {th }}$ row vector of the Laplacian ( or $H$ ) matrix.

From (1), one can get

$$
\begin{aligned}
& {\left[\dot{x}_{1}-\dot{x}_{n}, \ldots, \dot{x}_{n-1}-\dot{x}_{n}\right]^{T} } \\
= & {\left[v_{1}-v_{n}, \ldots, v_{n-1}-v_{n}\right]^{T} } \\
= & {\left[0_{n-1}, I_{n-1}\right] z . }
\end{aligned}
$$

Now, combining (24) and (25), we can get

$$
\dot{z}(t)=\left[\begin{array}{cc}
0_{n-1} & I_{n-1} \\
-f \beta_{0_{\sigma(t-\Delta)}} H(t) & -f \beta_{1_{\sigma(t-\Delta)}} I_{n-1}
\end{array}\right] z(t), z\left(t_{0}\right)=z(0) .
$$

We further have

$$
\begin{aligned}
& {\left[\begin{array}{cc}
0_{n-1} & I_{n-1} \\
-f \beta_{0_{\sigma(t-\Delta)}} H(t) & -f \beta_{1_{\sigma(t-\Delta)}} I_{n-1}
\end{array}\right] } \\
= & {\left[\begin{array}{cc}
I_{n-1} & 0_{n-1} \\
0_{n-1} & f I_{n-1}
\end{array}\right]\left[\begin{array}{cc}
0_{n-1} & I_{n-1} \\
-\beta_{0} I_{n-1} & -\beta_{1} I_{n-1}
\end{array}\right]_{\sigma(t-\Delta)} } \\
& \times\left[\begin{array}{cc}
H & 0_{n-1} \\
0_{n-1} & I_{n-1}
\end{array}\right]_{\sigma(t)} \\
\triangleq & F K_{\sigma(t-\Delta)} B_{a \sigma(t)},
\end{aligned}
$$

where $f$ is a scalar, and $f_{\text {diag }}$ denotes the diagonal matrix with diagonal elements. The equation (18) can now be proved.

According to the definition of $z(t)$, the consensus problem can be solved if the system (18) is globally uniformly asymptotically stable. This completes the proof of this theorem.

Remark 3: For the case without a controller fault or an uncertain parameter, it can be denoted by $f=1$. From the proof of Theorem 1, we can see that Theorem 1 is also applicable to the situations without a controller fault.

\subsection{Consensus with a controller fault}

For the multi-agent systems with a controller fault represented by the uncertain parameter, we only know the range of $f$. In order to solve the asynchronous switching with an uncertain parameter $f$, we rewrite the formation of $f$.

We define $F_{u} \triangleq \operatorname{diag}\left(1, \cdots, 1, f_{u}, \cdots f_{u}\right), \quad F_{d} \triangleq$ $\operatorname{diag}\left(1, \cdots, 1, f_{d}, \cdots f_{d}\right), F_{0} \triangleq \frac{1}{2}\left(F_{u}+F_{d}\right), F_{1} \triangleq \frac{1}{2}\left(F_{u}-F_{d}\right)$, then

$$
F=F_{0}+E F_{1},
$$

where $E=\operatorname{diag}(1 \cdots 1, e, \cdots, e)$, and $-1 \leqslant e \leqslant 1$.

Systems (18) can be replaced with

$$
\begin{aligned}
\dot{z}(t) & =F K_{\sigma(t-\Delta)} B_{a \sigma(t)} z(t) \\
& =\left(F_{0}+E F_{1}\right) K_{\sigma(t-\Delta)} B_{a \sigma(t)} z(t) \\
& =F_{0} K_{\sigma(t-\Delta)} B_{a \sigma(t)} z(t)+E F_{1} K_{\sigma(t-\Delta)} B_{a \sigma(t)} z(t) \\
& =\bar{A}_{\sigma(t-\Delta)} z(t),
\end{aligned}
$$

where $\bar{A}_{\sigma(t-\Delta)}=F_{0} K_{\sigma(t-\Delta)} B_{a \sigma(t)} z(t)+E F_{1} K_{\sigma(t-\Delta)} B_{a \sigma(t)} z(t)$.

From (29) and the inequality in Lemma 2, we now have the following theorem:

Theorem 2: For the given constants $\lambda_{p}>0, \mu_{p}>1$, and the multi-agent systems with switched topologies $\mathcal{G}_{p}$, $p \in \mathcal{M}, \forall\left(\sigma\left(t_{i}\right)=p, \sigma\left(t_{i}^{-}\right)=q\right) \in \mathcal{M} \times \mathcal{M}, p \neq q$, if there exist symmetric matrices $P_{p}>0$, positive constants $\varepsilon_{p}$, and $\varepsilon_{p q}, \forall p \in \mathcal{M}$, such that

$$
\left[\begin{array}{ccc}
D_{p} & \varepsilon_{p} F_{1} & P_{p}\left(K_{p} B_{a p}\right)^{T} \\
\varepsilon_{p} F_{1}^{T} & -\varepsilon_{p} I_{n-1} & 0_{n-1} \\
K_{p} B_{a p} P_{p} & 0_{n-1} & -\varepsilon_{p} I_{n-1}
\end{array}\right]<0
$$

where $D_{p}=\left(F_{0} K_{p} B_{a p}\right) P_{p}+P_{p}\left(F_{0} K_{p} B_{a p}\right)^{T}+\lambda_{p} P_{p}$, and

$$
\begin{aligned}
& P_{p}^{-1} \leq \mu_{p} P_{q}^{-1} \\
& {\left[\begin{array}{ccc}
\bar{D}_{p} & \varepsilon_{p q} F_{1} & P_{p}\left(K_{q} B_{a p}\right)^{T} \\
\varepsilon_{p q} F_{1}^{T} & -\varepsilon_{p q} I_{n-1} & 0_{n-1} \\
K_{q} B_{a p} P_{p} & 0_{n-1} & -\varepsilon_{p q} I_{n-1}
\end{array}\right]<0,}
\end{aligned}
$$

where $\bar{D}_{p}=\left(F_{0} K_{q} B_{a p}\right) P_{p}+P_{p}\left(F_{0} K_{q} B_{a p}\right)^{T}-\alpha_{p} P_{p}$, then the multi-agent systems (1) under consensus algorithm (2) with the fault tolerance (3) will reach a consensus with MDADT

$$
\tau_{a p}>\tau_{a p}^{*}=\frac{\Delta_{p_{-} \max }\left(\lambda_{P}+\alpha_{p}\right)+\ln \mu_{p}}{\lambda_{p}} .
$$

Proof: Based on the Schur complement lemma, one can see that the system (30) is equivalent to

$$
D_{p}-S_{p}^{T}\left[\begin{array}{cc}
-\varepsilon_{p} I_{n-1} & 0_{n-1} \\
0_{n-1} & -\varepsilon_{p} I_{n-1}
\end{array}\right]^{-1} S_{p}<0,
$$

where $S_{p}=\left[\begin{array}{c}\varepsilon_{p} F_{1}^{T} \\ \left(K_{p} B_{a p}\right) P_{p}\end{array}\right]$. This can be further written as

$$
\begin{aligned}
& D_{p}-S_{p}^{T}\left[\begin{array}{cc}
-\varepsilon_{p} I_{n-1} & 0_{n-1} \\
0_{n-1} & -\varepsilon_{p} I_{n-1}
\end{array}\right]^{-1} S_{p} \\
= & D_{p}+S_{p}^{T}\left[\begin{array}{cc}
\varepsilon_{p}{ }^{-1} I_{n-1} & 0_{n-1} \\
0_{n-1} & \varepsilon_{p}^{-1} I_{n-1}
\end{array}\right] S_{p} \\
= & D_{p}+\left[\begin{array}{c}
F_{1} \\
\varepsilon_{p}{ }^{-1} P_{p}\left(K_{p} B_{a p}\right)^{T}
\end{array}\right] S_{p} \\
= & D_{p}+\varepsilon_{p} F_{1} F_{1}^{T}+\varepsilon_{p}{ }^{-1} P_{p}\left(K_{p} B_{a p}\right)^{T}\left(K_{p} B_{a p}\right) P_{p} \\
< & 0 .
\end{aligned}
$$

According to Lemma 2, one can get

$$
D_{p}+F_{1} E\left(K_{p} B_{a p}\right) P_{p}+P_{p}\left(K_{p} B_{a p}\right)^{T} E^{T} F_{1}^{T}<0 .
$$

This can also be further represented as

$$
\begin{aligned}
& D_{p}+F_{1} E\left(K_{p} B_{a p}\right) P_{p}+P_{p}\left(K_{p} B_{a p}\right)^{T} E^{T} F_{1}^{T} \\
= & \left(F_{0} K_{p} B_{a p}+F_{1} E K_{p} B_{a p}\right) P_{p} \\
& +P_{p}\left(F_{0} K_{p} B_{a p}+F_{1} E\left(K_{p} B_{a p}\right)\right)^{T}+\lambda_{p} P_{p}
\end{aligned}
$$


Using $\operatorname{diag}\left\{P_{p}^{-1}\right\}$ to pre- and post-multiply both sides of systems (36), one has

$$
\begin{aligned}
& P_{p}^{-1}\left(F_{0} K_{p} B_{a p}+F_{1} E K_{p} B_{a p}\right) \\
& +\left(F_{0} K_{p} B_{a p}+F_{1} E K_{p} B_{a p}\right)^{T} P_{p}{ }^{-1}+\lambda_{p} P_{p}{ }^{-1} \\
& <0 .
\end{aligned}
$$

Denote $\bar{P}_{p}=P_{p}{ }^{-1}$. We can also see that $\bar{P}_{p}>0$ and systems (37) are equivalent to

$$
\begin{aligned}
& \bar{P}_{p}\left(F_{0} K_{p} B_{a p}+F_{1} E K_{p} B_{a p}\right)+\left(F_{0} K_{p} B_{a p}+F_{1} E K_{p} B_{a p}\right) \bar{P}_{p} \\
& +\lambda_{p} \bar{P}_{p}<0 .
\end{aligned}
$$

Based on systems (29) and (38), one has

$$
\bar{P}_{p}\left(F K_{p} B_{a p}\right)+\left(F K_{p} B_{a p}\right)^{T} \bar{P}_{p}<-\lambda_{p} \bar{P}_{p} .
$$

Similarly, we can have

$$
\bar{P}_{p}\left(F K_{q} B_{a p}\right)+\left(F K_{q} B_{a p}\right)^{T} \bar{P}_{p}<\alpha_{p} \bar{P}_{p} .
$$

From (31), one has

$$
\bar{P}_{p} \leq \mu_{p} \bar{P}_{q}
$$

Combining the results in (39), (40) and (41) and Lemma 1 , we can then establish the results in Theorem 2. The proof is completed.

Remark 4: Theorem 2 is based on Lemma 1 and on the idea that the topologies changing replaces subsystem switching. Hence the results on MDADT and switched systems are also applicable to the multi-agent systems under switched topologies.

Using a similar process, we can establish and prove Corollary 1 below when using a common $P$ to replace $P_{p}$.

Corollary 1: For the multi-agent systems with switched topologies $\mathcal{G}_{p}, p \in \mathcal{M}, \forall\left(\sigma\left(t_{i}\right)=p, \sigma\left(t_{i}^{-}\right)=q\right) \in$ $\mathcal{M} \times \mathcal{M}, p \neq q$, if there exist a symmetric matrix $P>0$, positive constants $\varepsilon_{p}, \forall p \in \mathcal{M}$, such that

$$
\left[\begin{array}{ccc}
D_{p} & \varepsilon_{p} F_{1} & P\left(K_{p} B_{a p}\right)^{T} \\
\varepsilon_{p} F_{1}^{T} & -\varepsilon_{p} I_{n-1} & 0_{n-1} \\
\left(K_{p} B_{a p}\right) P & 0_{n-1} & -\varepsilon_{p} I_{n-1}
\end{array}\right]<0
$$

where $D_{p}=\left(F_{0} K_{p} B_{a p}\right) P+P\left(F_{0} K_{p} B_{a p}\right)^{T}+\lambda_{p} P$,

$$
\left[\begin{array}{ccc}
\bar{D}_{p} & \varepsilon_{p q} F_{1} & P\left(K_{q} B_{a p}\right)^{T} \\
\varepsilon_{p q} F_{1} T & -\varepsilon_{p q} I & 0_{n-1} \\
K_{q} B_{a p} P & 0_{n-1} & -\varepsilon_{p q} I_{n-1}
\end{array}\right]<0
$$

where $\bar{D}_{p}=\left(F_{0} K_{q} B_{a p}\right) P+P\left(F_{0} K_{q} B_{a p}\right)^{T}-\alpha_{p} P$, then the multi-agent systems (1) under consensus algorithm (2) with the fault tolerance (3) will reach the consensus finally with MDADT

$$
\tau_{a p}>\tau_{a p}^{*}=\frac{\Delta_{p_{-} \max }\left(\lambda_{P}+\alpha_{p}\right)}{\lambda_{p}} .
$$

Remark 5: MDADT is used in the proof for reaching a consensus when the multi-agent systems are switching. In order to guarantee the condition of MDADT, the statistical information on the dwell time under each topology is required to confirm that the average dwell time is longer than the offset.

\subsection{Extension to Synchronous Switching}

One can see that if the time lag $\Delta(t)=0$, the asynchronous switching is replaced with synchronous switching in the algorithm (2).

In the synchronous switching situation, systems (2) change to

$u_{i}(t)=f \beta_{0_{\sigma(t)}} \sum_{j \in \mathcal{N}_{\mathrm{i}}(\mathrm{t})} a_{i j_{\sigma(t)}}\left(x_{j}(t)-x_{i}(t)\right)-f \beta_{1_{\sigma(t)}} v_{i}(t)$.

Likewise, the following corollaries can be obtained for the case of synchronous switching multi-agent systems.

Corollary 2: For the given constants $\lambda_{p}>0, \mu_{p}>1$, and the switched topologies $\mathcal{G}_{p}$ of the multi-agent systems, $p \in \mathcal{M}, \forall\left(\sigma\left(t_{i}\right)=p, \sigma\left(t_{i}^{-}\right)=q\right) \in \mathcal{M} \times \mathcal{M}, p \neq q$, if there exist symmetric matrices $P_{p}>0$, positive constants $\varepsilon_{p}$, $\forall p \in \mathcal{M}$, such that

$$
\left[\begin{array}{ccc}
D_{p} & \varepsilon_{p} F_{1} & P_{p}\left(K_{p} B_{a p}\right)^{T} \\
\varepsilon_{p} F_{1}^{T} & -\varepsilon_{p} I_{n-1} & 0_{n-1} \\
K_{p} B_{a p} P_{p} & 0_{n-1} & -\varepsilon_{p} I_{n-1}
\end{array}\right]<0
$$

where $D_{p}=\left(F_{0} K_{p} B_{a p}\right) P_{p}+P_{p}\left(F_{0} K_{p} B_{a p}\right)^{T}+\lambda_{p} P_{p}$,

$$
P_{p}^{-1} \leq \mu_{p} P_{q}^{-1}
$$

then the multi-agent systems (1) under consensus algorithm (45) with the fault tolerance (3) reach the consensus when

$$
\tau_{a p}>\tau_{a p}^{*}=\frac{\ln \mu_{p}}{\lambda_{p}}
$$

Corollary 3: For the switched topologies $\mathcal{G}_{p}$ of the multi-agent systems, $p \in \mathcal{M}$, if there exist a symmetric matrix $P>0$, positive constants $\varepsilon_{p}, \forall p \in \mathcal{M}$, such that

$$
\left[\begin{array}{ccc}
D_{p} & \varepsilon_{p} F_{1} & P\left(K_{p} B_{a p}\right)^{T} \\
\varepsilon_{p} F_{1}^{T} & -\varepsilon_{p} I_{n-1} & 0_{n-1} \\
\left(K_{p} B_{a p}\right) P & 0_{n-1} & -\varepsilon_{p} I_{n-1}
\end{array}\right]<0
$$

where $D_{p}=\left(F_{0} K_{p} B_{a p}\right) P+P\left(F_{0} K_{p} B_{a p}\right)^{T}+\lambda_{p} P$, then the multi-agent systems (1) under consensus algorithm (45) with the fault tolerance (3) will reach the consensus finally.

Remark 6: LMIs toolbox in MATLAB is often used to find proper matrices $P$ and constants $\varepsilon$. Although the existence of proper matrices and constants is the sufficient condition, the results in this paper provide a guarantee on the consensus under asynchronous switching, especially when the systems are with an uncertain parameter. 
Since there are many nonlinear cases and TakagiSugeno (T-S) fuzzy model has been popularly utilized in representing nonlinear systems [31-33], one of our future works is to investigate the coordination of the nonlinear multi-agent systems under asynchronous switching.

\section{NUMERICAL EXAMPLE}

In this section, numerical results are presented to demonstrate the potential and validity of our developed theoretical results. For this example, the second-order multi-agent systems are with 4 agents and the topologies are switched. Through this numerical example, the asynchronous switching's reaction to a consensus can also be seen clearly. In the same set-up, the corresponding validation can be done for the common $P$ case and the synchronous switching cases. This is omitted due to space limitations.

Consider the adjacency matrices of the two considered topologies described by

$$
\left[\begin{array}{llll}
0 & 0 & 0 & 0 \\
1 & 0 & 0 & 0 \\
0 & 1 & 0 & 0 \\
1 & 0 & 0 & 0
\end{array}\right] \text {, and }\left[\begin{array}{cccc}
0 & 0 & 1 & 0 \\
1 & 0 & 0 & 0 \\
0 & 1 & 0 & 1 \\
1 & 0 & 0 & 0
\end{array}\right]
$$

The two-level signal values (low and high) of switching signals $\sigma(t)$ stand for these two topologies. The purpose here is to show the influence of asynchronous switching and present the tendency of states and velocities of agents when the dwell times satisfy the corresponding conditions (8). In order to achieve this, the changing of energy function (Lyapunov function) is shown in Fig.1, and states and velocities are shown in Fig.2., when switching signals $\sigma(t)$ and $\sigma^{\prime}(t)$ change.

Selection of the parameters for the system model and the controller is based on the physical meanings of parameters. The sign $\mu$ stands for the jumping strength when topologies change. The symbols $\lambda$ and $\alpha$ denote the convergence rate and the divergence speed. Changing these parameters will affect constrained conditions which results in the changing of the average dwell time. The looser the constrained conditions are, the lager the average dwell time is.

The maximal delay of asynchronous switching of the first topology is 0 , and the maximal delay of asynchronous switching of the second topology is 6 seconds. In this example, we just know the range $0.9 \leq f \leq 1.1$, i.e., $f_{d}=0.9$ and $f_{u}=1.1$. By providing proper constants $\lambda_{1}=0.020$, $\mu_{1}=1.88, \alpha_{1}=0, \lambda_{2}=0.183, \mu_{2}=2.47, \alpha_{2}=0.845$, the LMIs toolbox can find proper solutions and get the proper dwell time satisfying conditions (8). The simulation results are shown in Fig.1. and Fig.2.

At the running time slots [0,1.730), [8.04,9.77), $[15.65,19.74)$ and $[25.47,34.58)$, the network is synchronous, the energy function in Fig.1. drops down quickly except for at the topologies switching time spot, the states and velocities of agents in Fig.2. are reaching a consensus. At the running time slots [1.73,8.04), $[9.77,15.65),[19.74,25.47)$ and $[34.58,40.37)$, the network is asynchronous, the energy function in Fig.1. increases quickly, the states and velocities of agents in Fig.2. tend to diverge. When the dwell times meet the conditions (8), the consensus is reached.

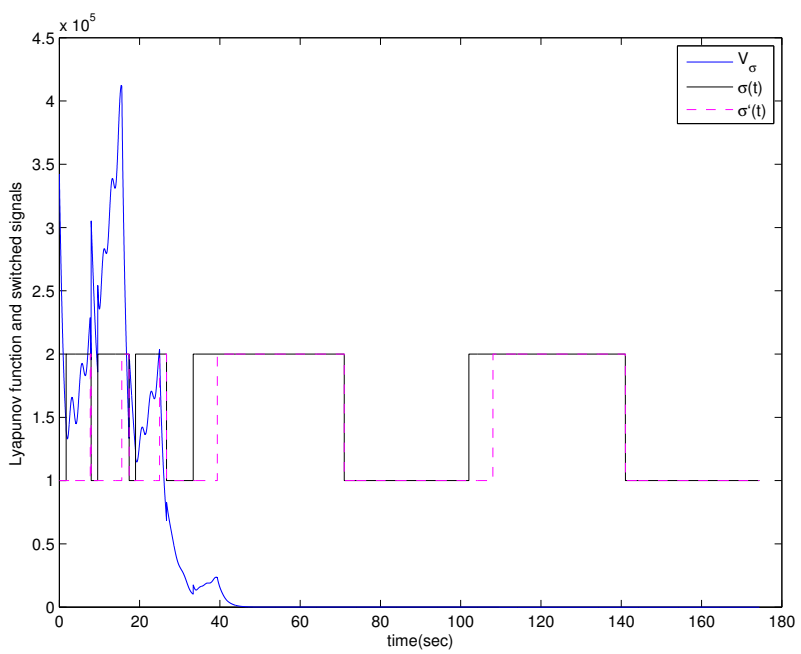

Fig. 1. The changing of the Lyapunov function and switched signals
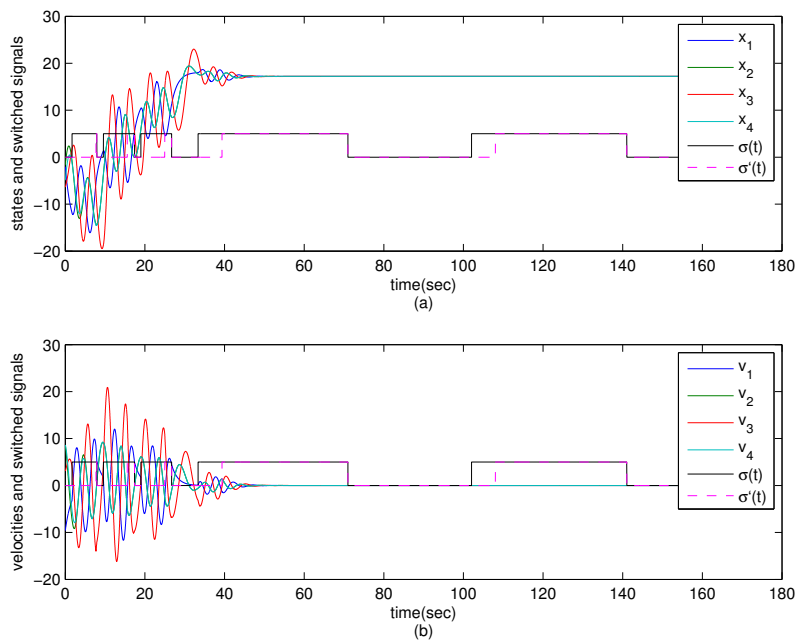

Fig. 2. The changing of the states, velocities and switched signals

\section{CONCLUSIONS}

The consensus of the second-order multi-agent systems under asynchronous switching with a controller fault is in- 
vestigated. We analyzed the relationship between consensus and steadiness, and prove the consensus, and provided conditions for the consensus, and also extended the results to the synchronous switching multi-agent systems. Simulation results clearly demonstrate the asynchronous switching's reaction to a consensus. With both analytical and numerical results, we show that a consensus under asynchronous switching with a controller fault can also be reached under proper conditions despite the reaction of asynchronous switching. The methods developed in this paper can also be potentially applied to study other problems such as containment control and vehicle formations in the presence of asynchronous switching for the underlying systems.

\section{REFERENCES}

[1] Y. Yuan, H. Yuan, Z. Wang, L. Guo, and H. Yang, “Optimal control for networked control systems with disturbances: a delta operator approach," IET Control Theory and Applications, vol. 11, no. 9, pp. 1325-1332, 2017.

[2] J. Ota, "Multi-agent robot systems as distributed autonomous systems," Advanced engineering informatics, vol. 20, no. 1, pp. 59-70, 2006.

[3] M. Zhang, P. Tian, and X. Chen, "Unmanned aerial vehicle guidance law for ground target circumnavigation using range-based measurements," International Journal of Control, Automation and Systems, vol. 15, no. 5, pp. 24552460, 2017.

[4] R. Olfati-Saber and R. M. Murray, "Consensus problems in networks of agents with switching topology and timedelays," IEEE Transactions on automatic control, vol. 49, no. 9, pp. 1520-1533, 2004.

[5] S.-X. Tang, J. Qi, and J. Zhang, "Formation tracking control for multi-agent systems: A wave-equation based approach," International Journal of Control, Automation and Systems, vol. 15, no. 6, pp. 2704-2713, 2017.

[6] B. Zhu, C. Meng, and G. Hu, "Robust consensus tracking of double integrator dynamics by bounded distributed control," International Journal of Robust and Nonlinear Control, vol. 26, no. 7, pp. 1489-1511, 2016.

[7] D. Zheng, H. Zhang, J. Zhang and G. Wang, "Consensus of multi-agent systems with faults and mismatches under switched topologies using a delta operator method," Neurocomputing, 2018. DOI: 10.1016/j.neucom.2018.07.017

[8] D. Ma and Y. Sun, "Finite-time circle surrounding control for multi-agent systems," International Journal of Control, Automation and Systems, vol. 15, no. 4, pp. 1536-1543, 2017.

[9] B. Li, Z.-Q. Chen, C.-Y. Zhang, Z.-X. Liu, and Q. Zhang, "Containment control for directed networks multi-agent system with nonlinear dynamics and communication timedelays," International Journal of Control, Automation and Systems, pp. 1-8, 2017.
[10] D. Zheng and H. Zhang, "Research on the transformation of control protocols among three kinds of cooperative control for multi-agent systems," in 2016 8th International Conference on Intelligent Human-Machine Systems and Cybernetics (IHMSC), pp. 301-304, IEEE, 2016.

[11] W. Liu and J. Huang, "Adaptive leader-following consensus for a class of higher-order nonlinear multi-agent systems with directed switching networks," Automatica, vol. 79, pp. 84-92, 2017.

[12] R. Olfati-Saber, J. A. Fax, and R. M. Murray, "Consensus and cooperation in networked multi-agent systems," Proceedings of the IEEE, vol. 95, no. 1, pp. 215-233, 2007.

[13] T. Li and J.-F. Zhang, "Mean square average-consensus under measurement noises and fixed topologies: Necessary and sufficient conditions," Automatica, vol. 45, no. 8, pp. 1929-1936, 2009.

[14] W. Ren and R. W. Beard, "Consensus seeking in multiagent systems under dynamically changing interaction topologies," IEEE Transactions on automatic control, vol. 50, no. 5, pp. 655-661, 2005.

[15] W. Yu, G. Chen, and M. Cao, "Some necessary and sufficient conditions for second-order consensus in multi-agent dynamical systems," Automatica, vol. 46, no. 6, pp. 10891095, 2010.

[16] W. Hou, M. Fu, H. Zhang, and Z. Wu, "Consensus conditions for general second-order multi-agent systems with communication delay," Automatica, vol. 75, pp. 293-298, 2017.

[17] Y. Huang and Y. Jia, "Fixed-time consensus tracking control for second-order multi-agent systems with bounded input uncertainties via nfftsm," IET Control Theory and Applications, vol. 11, no. 16, pp. 2900-2909, 2017.

[18] C. Song, J. Cao, and Y. Liu, "Robust consensus of fractional-order multi-agent systems with positive real uncertainty via second-order neighbors information," Neurocomputing, vol. 165, pp. 293-299, 2015.

[19] X. Wu, Y. Tang, J. Cao, and W. Zhang, "Distributed consensus of stochastic delayed multi-agent systems under asynchronous switching," IEEE transactions on cybernetics, vol. 46, no. 8, pp. 1817-1827, 2016.

[20] F. Xiao and L. Wang, "Asynchronous consensus in continuous-time multi-agent systems with switching topology and time-varying delays," IEEE Transactions on Automatic Control, vol. 53, no. 8, pp. 1804-1816, 2008.

[21] L. Fang and P. J. Antsaklis, "Asynchronous consensus protocols using nonlinear paracontractions theory," IEEE Transactions on Automatic Control, vol. 53, no. 10, pp. 2351-2355, 2008.

[22] L. Lamport, "Lower bounds for asynchronous consensus," Distributed Computing, vol. 19, no. 2, pp. 104-125, 2006.

[23] Q. Zheng and H. Zhang, "Asynchronous $h_{\infty}$ fuzzy control for a class of switched nonlinear systems via switching fuzzy lyapunov function approach," Neurocomputing, vol. 182, pp. 178-186, 2016.

[24] L. Zhang and H. Gao, "Asynchronously switched control of switched linear systems with average dwell time," Automatica, vol. 46, no. 5, pp. 953-958, 2010. 
[25] D. Zheng, H. Zhang, and Q. Zheng, "Consensus analysis of multi-agent systems under switching topologies by a topology-dependent average dwell time approach," IET Control Theory and Applications, vol. 3, no. 3, pp. 429438, 2017.

[26] R. A. Horn and C. R. Johnson, Matrix analysis. Cambridge university press, 1990.

[27] W. He and J. Cao, "Consensus control for high-order multi-agent systems," IET Control Theory and Applications, vol. 5, no. 1, pp. 231-238, 2011.

[28] Y. Cao and Y. Sun, "Consensus analysis for third-order multiagent systems in directed networks," Mathematical Problems in Engineering, vol. 2015, 2015.

[29] X. Zhao, L. Zhang, P. Shi, and M. Liu, "Stability and stabilization of switched linear systems with mode-dependent average dwell time," IEEE Transactions on Automatic Control, vol. 57, no. 7, pp. 1809-1815, 2012.

[30] L. Xie, "Output feedback protect $h_{\infty}$ control of systems with parameter uncertainty," International Journal of Control, vol. 63, no. 4, pp. 741-750, 1996.

[31] H. Li, J. Wang, H. Du, and H. R. Karimi, "Adaptive sliding mode control for takagi-sugeno fuzzy systems and its applications," IEEE Transactions on Fuzzy Systems, vol. 26, no. 2, pp. 531-542, 2018.

[32] Q. Zhou, H. Li, L. Wang, and R. Lu, "Prescribed performance observer-based adaptive fuzzy control for nonstrictfeedback stochastic nonlinear systems," IEEE Transactions on Systems, Man, and Cybernetics: Systems, 2017. DOI: 10.1109/TSMC.2017.2738155

[33] Q. Zhou, L. Wang, C. Wu, and H. Li, "Adaptive fuzzy tracking control for a class of pure-feedback nonlinear systems with time-varying delay and unknown dead zone," Fuzzy Sets and Systems, vol. 329, pp. 36-60, 2017.

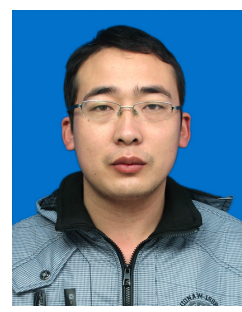

Dianhao Zheng received the B.Eng. degree in electronic science and technology from China University of Mining and Technology, Xuzhou, in 2009, and the M.S. degree in circuits and systems from University of Electronic Science and Technology of China, Chengdu, in 2012. He is currently working towards two Ph.D. degrees in circuits and systems at the University of Electronic Science and Technology of China and in engineering and information technology at the University of Technology Sydney. He was an engineer from 2012 to 2014. His research interests include cooperative control, multi-agent systems, and switched systems.

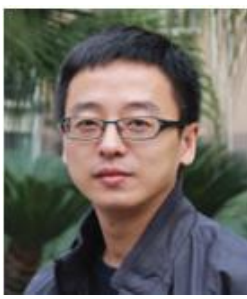

Hongbin Zhang received the B.Eng. degree in aerocraft design from Northwestern Polytechnical University, Xian, China, in 1999, and the MEng and $\mathrm{PhD}$ degrees in circuits and systems from the University of Electronic Science and Technology of China, Chengdu, in 2002 and 2006, respectively. He has been with the School of Electrical Engineering, University of Electronic Science and Technology of China, since 2002, where he is currently a professor. From August 2008 to August 2010, he has served as a research fellow with the Department of Manufacturing Engineering and Engineering Management, City University of Hong Kong, Kowloon, Hong Kong. His current research interests include intelligent control, autonomous cooperative control and integrated navigation.

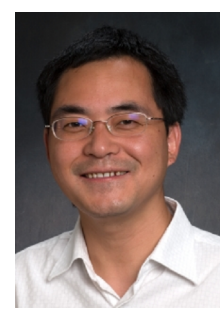

J. Andrew Zhang received the B.Sc. degree from Xi'an JiaoTong University, China, in 1996, the M.Sc. degree from Nanjing University of Posts and Telecommunications, China, in 1999, and the Ph.D. degree from the Australian National University, in 2004. Currently, Dr. Zhang is an associate Professor in School of Computing and Communications, University of Technology Sydney, Australia. He was a researcher with Data 61, CSIRO, Australia from 2010 to 2016, the Networked Systems, NICTA, Australia from 2004 to 2010, and ZTE Corp, Nanjing, China from 1999 to 2001. Dr. Zhang's research interests are in the area of signal processing for wireless communications and sensing, and autonomous vehicular networks. He has won 4 best paper awards for his work. He is a recipient of CSIRO Chairman's Medal and the Australian Engineering Innovation Award in 2012 for exceptional research achievements in multi-gigabit wireless communications.

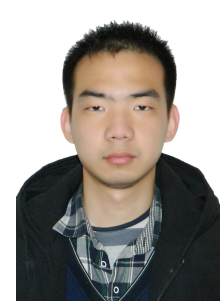

Yang Li received the B.E. degree in electronic and information engineering and M.S. degree in circuits and systemsfrom University of Electronic Science and Technology of China, Chengdu, in 2014 and 2017, respectively. He is currently working towards his Ph.D. degree in circuits and systems at the University of Electronic Science and Technology of China, Chengdu. His research interests include fuzzy control and switched systems. 